\title{
Les indications médicales de la fécondation in vitro
}

Les indications de la fertilisation in vitro se sont aujourd'hui considérablement élargies, permettant de faire passer cette méthode au rang des techniques majeures de lutte contre les stérilités masculines et féminines.

\section{René Frydman \\ Professeur agrégé à l'université Paris-Sud.}

\section{Isu Eibschitz}

Chef de l'unité d'infertilité mécanique au Centre médical Haïfa (Rothschild). Docent à la Faculté de médecine, Technion, Haïfa, Israël.

\section{Emile Papiernik}

Professeur à l'université ParisSud. Chef $d u$ service de gynécologie-obstétrique de l'hôpital Antoine-Béclère. Directeur de l'unité 187 de l'Inserm.

\section{ADRESSE}

E. Papiernik, R. Frydman : Hôpital AntoineBéclère, 157, rue de la porte de Trivaux. 92141 Clamart.

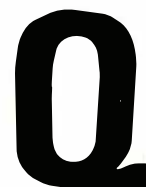
ue les deux cellules de la reproduction sperme et ovule quittent le corps humain pour se rencontrer et s'assembler avant de regagner le lieu naturel de la gestation est une révolution qui marquera la deuxième partie du $\mathrm{xx}^{\mathrm{e}}$ siècle. L'ère de la maîtrise de la reproduction par l'homme ouvre de grandes perspectives. La technique de fécondation in vitro et de transfert d'embryon (FIVETE) a été mise au point en 1978 [I], elle est depuis en constante évolution. Cette méthode a été utilisée de façon privilégiée pour les femmes présentant des stérilités mécaniques d'origine tubaire (trompes obturées, absentes ou altérées). Mais très vite on a voulu élargir son utilisation à des stérilités d'origine masculine, idiopathique, immunologique ou d'autres encore $[2,3,4,5,6]$. Cette technique autorise l'intervention d'un gamète féminin étranger : dons d'ovocytes, dons d'embryons sont désormais possibles $[7,8,9]$. Ces cas de figure mettent en jeu de nombreux problèmes éthiques actuellement discutés.

\section{Bilan préalable}

Les patientes candidates à une fécondation in vitro subissent un bilan préalable qui prend en compte :

leur âge, en effet la fécondité naturelle baisse après 35 ans, les résul- tats de la fécondation in vitro montrent une chute des pourcentages après 40 ans $(7,2 \%$ au-dessus de 40 ans, contre $12,5 \%$ pour les moins de 35 ans, Congrès d'Helsinki, mai I984). Le taux de fausses-couches est également augmenté après 40 ans;

leurs antécédents médicaux et chirurgicaux : recherche d'une salpingite, de suites compliquées d'une interruption volontaire de grossesse; utilisation et conséquences d'un dispositif intra-utérin, existence d'une gonorrhée, d'une tuberculose génitale;

leur passé obstétrical : faussecouche spontanée ou accouchement. La plupart de ces patientes ont subi sans succès une ou plusieurs opérations de microchirurgie des trompes ou bien des salpingectomies bilatérales pour grossesses extra-utérines ou pour inflammation sévère des trompes.

Les investigations suivantes sont nécessaires avant la tentative de fécondation in vitro :

(a) l'exploration de l'ovulation : sa qualité peut être appréciée par l'examen des courbes ménothermiques et des dosages hormonaux d'estradiol, de progestérone, de prolactine et de la série androgénique si besoin;

(b) l'étude du sperme : spermogramme, spermocytogramme et spermoculture ;

(c) l'étude de la cavité utérine : par hystérographie ou, plus récemment, par hystéroscopie qui permet 


\section{REFERENCES}

I. Steptoe PC, Edwards RG. Birth after reimplantation of a human embryo. Lancet 1978; 2 : 366.

2. Edwards RG, Fishel SB, Cohen J, et al. Factors influencing the success of in vitro fertilization for alleviating human infertility. 7 In Vitro Fertil Embr Transf 1984; $1: 3-23$.

3. Trouson A, Wood C. Extracorporeal fertilization and embryo transfer. Clin Obstet Gynaecol I98I; $8: 68 \mathrm{I}-713$.

4. Mahadevan MM, Trouson AO, Leeton JF. The relationship of tubal blockage, infertility of unknown cause, suspected male infertility, and endometriosis to success of in vitro fertilization and embryo transfer. Fertil Steril 1983; 40 : 75562.

5 Trounson A, Wood C. In vitro fertilization results, 1979-1982, at Monash University, Queen Victoria, and EP Worth Medical Centers. 7 In Vitro Fertil Embr Transf 1984; I : 42-7.

6. Jones HW, JR, Acosta AA, Andrews MC, et al. What is a pregnancy? A question for programs of in vitro fertilization. Fertil Steril 1983; $40: 728$ 33 .

7. Wood $C$, Trounson $A$. In vitro fertilization and embryo transfer. In: Bonnar J, Ed. Recent advances in obstetrics and gynaecology, $n^{\circ} 14$. London: Churchill Livingstone, $1982: 259-88$.

8. Trounson A, Leeton J, Besanko M, Wood C, Conti A. Pregnancy established in an infertile patient af ter transfer of a donated embryo fertilized in vitro. $\mathrm{Br}$ Med 7 1983; $286: 835-88$.

9. Trounson A, Mohr L. Human pregnancy following cryopreservation, thawing and transfer of eight-cell embryo. Nature 1983; $305:$ 707-9.

10. Frydman R, Feinstein MC, Testart J, et al Recueil d'ovocytes humains en phase ovulatoire. f Gyn Obst Biol Reprod 1981; 10 : 127-32.

II. Bclaisch-Allart JC, Hazout A, Guillet-Rosso F, Glissant M, Testart J, Frydman R. Various techniques for ovocyte recovery in an IVF and ET program. 7 In vitro Fertil Embr Transf 1985; d'explorer la cavité utérine et surtout la qualité de la muqueuse qui la tapisse. Cet examen permet, de plus, de préciser les orifices tubaires et surtout le canal cervical dont le défilé est important à connaître pour la qualité du replacement. Une hystérométrie sera d'ailleurs systématiquement pratiquée afin de connaître la distance idéale de dépose - de ou des - embryons lors du transfert;

(d) une coelioscopie de bilan est souvent nécessaire afin d'éliminer une contre-indication chirurgicale pelvienne et surtout afin de préciser l'accessibilité et la mobilité des ovaires qui permettra de décider du mode de recueil ovocytaire le mieux adapté chez cette patiente. En effet, le recueil d'ovocytes pendant la fécondation in vitro (FIV) peut être réalisé soit par coelioscopie sous anesthésie générale, soit par coelioscopie sous anesthésie locale, soit par ponction sous contrôle échographique trans-vésical percutané [Io, I I, I2], soit par ponction sous échographie par voie transvaginale [13]. Cette première phase d'exploration sera complétée par un examen gynécologique associant des frottis cervico-vaginaux, un examen des seins, un examen médical complet, et un examen sérologique de la rubéole, de la toxoplasmose et éventuellement des chlamydiae.

Initialement, seules les femmes atteintes de stérilité tubaire définitive pouvaient bénéficier de la fécondation in vitro, ces patientes étaient par le passé orientées vers l'adoption.

\section{Stérilités tubaires}

La stérilité tubaire reste aujourd'hui l'indication privilégiée de la FIVETE; dans la littérature ce groupe constitue plus de $50 \%$ des couples traités [5, I4]. Nombreuses sont les patientes de ce groupe qui présentent des adhérences intrapéritonéales recouvrant les ovaires. Des ovariolyses ou transpositions ovariennes ont été proposées afin de rendre les ovaires accessibles à la ponction. En cas de tuberculose génitale, la fécondation in vitro est le seul traitement possible car la microchirurgie est contre-indiquée dans de telles circonstances [ 15 ]. En présence de trompes altérées, on sait qu'il s'agit de stérilité tubaire relative, liée à des altérations de la paroi tubaire qui entravent le passage d'une ou des deux trompes soit à l'intérieur de celle-ci, soit à l'extérieur réalisant un phimosis plus ou moins serré gênant la capture de l'ovule par la trompe. La conservation d'une certaine perméabilité tubaire n'exclut pas la possibilité de survenue naturelle de grossesse (intra ou extra-utérine); nous avons d'ailleurs proposé une prise en charge par stimulation et monitorage échographique de ce groupe de patientes inscrites pour fécondation in vitro et présentant une ou deux trompes encore perméables et qui attendaient une place disponible. Sur 470 cycles traités sans fécondation in vitro : 46 grossesses ont été obtenues (4 grossesses extrautérines, 7 fausses-couches et 35 grossesses évolutives).

En cas d'altération tubaire, la fécondation in vitro peut être une solution retenue bien que non privilégiée en fonction de l'âge de la patiente, du nombre de grossesses extra-utérines et de la durée de la stérilité.

Le pourcentage de succès de la fécondation in vitro allant en augmentant, d'autres indications ont été successivement proposées.

\section{Stérilité masculine}

Le nombre de spermatozoïdes mobiles nécessaires pour réaliser la fécondation in vitro est très faible (moins de roo 000). De nombreux auteurs ont proposé d'utiliser ces techniques pour traiter les infertilités liées à des perturbations spermatiques : les indications masculines ont été individualisées, elles représentent 7 à $14 \%$ des indications selon les auteurs $[5,16,17]$. Les critères d'anormalité du spermogramme $(<20$ millions de spermatozoïdes $/ \mathrm{ml},<50 \%$ de mobilité, < $60 \%$ de morphologie normale) sont loin d'être une frontière absolue; de nomb̂reuses grossesses naturelles ont été obtenues avec des spermes déficients sur l'un ou l'autre de ces paramètres. Afin d'apprécier la fécondance du 


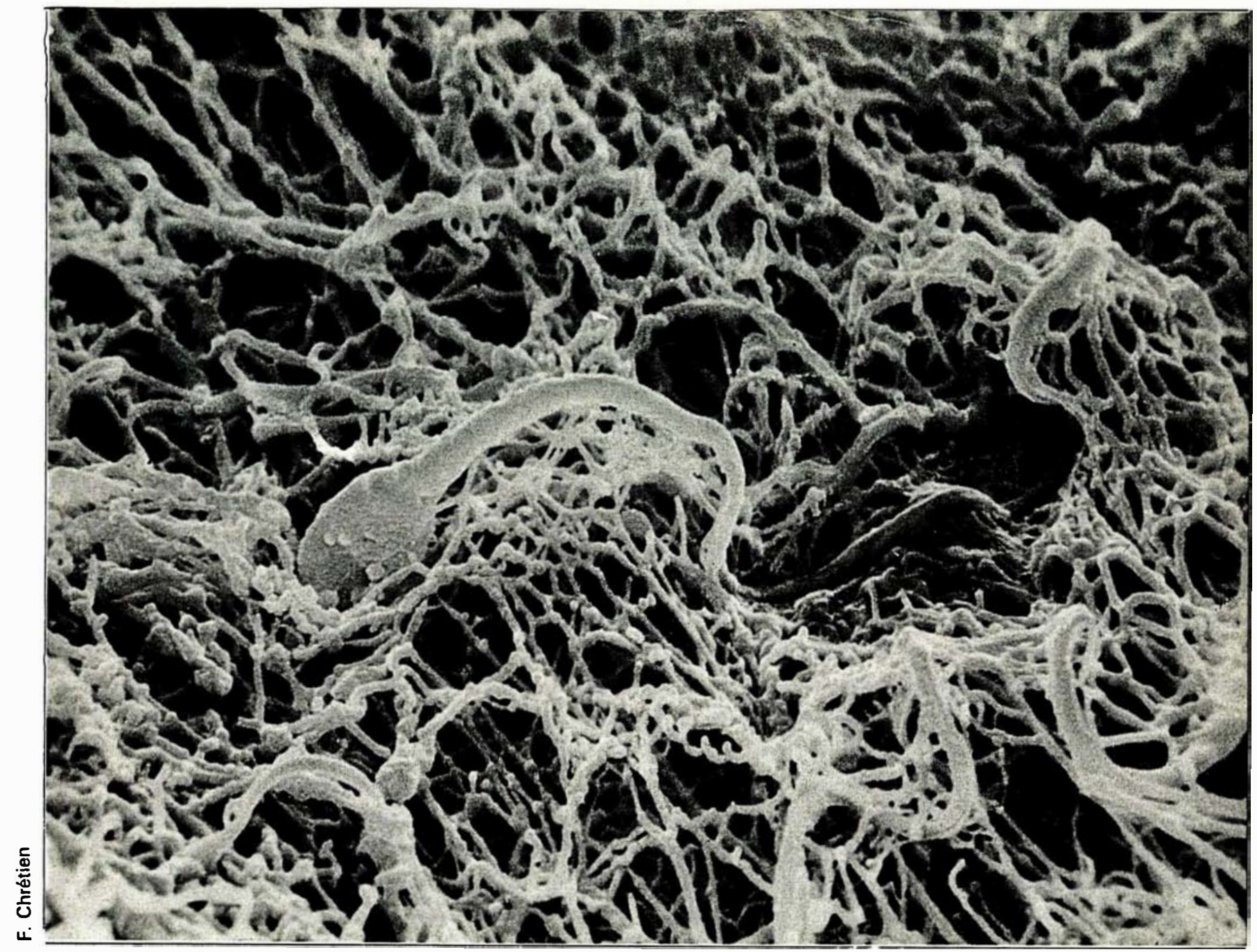

Réseau de filaments glycoprotéiques du mucus cervical au $28^{e}$ jour du cycle et spermatozoïde.

sperme, le test de l'ovocyte dépellucidé de hamster a été proposé mais a donné des résultats contradictoires $[4,18$, I9]. Le test de survie en milieu de fécondation a été proposé comme une nouvelle approche de la fécondance du sperme. S'il permet d'éliminer les cas où la survie des gamètes est nulle 24 heures après inoculation au milieu, il n'est pas assez précis pour refléter les capacités de fécondance du sperme considéré. Il est logique de penser qu'en déposant des spermatozoïdes directement sur l'ovocyte, on épargnerait à ceux-ci de parcourir le chemin que représente la montée de la filière génitale. L'avenir réside dans les différents types de préparation du sperme au laboratoire, mieux adaptée à chaque type d'anomalie $\mathrm{m} / \mathrm{s} \mathrm{mai} 85$ du sperme traité. Il existe un groupe d'infertilité masculine où une anomalie spécifique a pu être mise en évidence, telle la dyskinésie flagellaire, la présence d'auto-anticorps, l'altération ou l'absence de la cape post-acrosomiale; dans ces cas, l'intérêt de la fécondation in vitro est encore discuté. Une méthode d'avenir sera peut-être l'injection de spermatozoïdes sous la membrane pellucide de l'ovocyte sous contrôle microscopique.

Ainsi la définition des critères cliniques et biologiques de l'infertilité masculine justifiant le recours à la FIVETE est imprécise et variable selon les auteurs, si bien que le cadre est mal défini et qu'il n'y a pas d'étude prospective comparant d'un côté la fécondation in vitro, de l'au- tre des techniques d'insémination ou la fécondation naturelle.

Chez la femme, la présence d'anticorps anti-spermatozoïdes a été rendue responsable de l'infertilité. Ces anti-corps peuvent être présents dans le plasma ou au niveau du col utérin. Des variations de ces anticorps sont possibles chez la même patiente, ce qui explique la survenue de certaines grossesses spontanées.

\section{Stérilité immunologique}

Ces anticorps pourraient s'opposer au déplacement des spermatozoïdes ou bien agir au niveau de l'interaction entre le spermatozoïde et la membrane de l'ovocyte $[20,21]$. Les trois méthodes les plus fréquem- 


\section{REFERENCES}

12. Lenz S, Lauritsen JG. Ultrasonically guided percutaneous aspiration of human follicles under local anesthesia. A new method of collecting ovocytes for in vitro fertilization. Fertil Steril 1982 $3^{8}: 673-7$.

13. Dellenbach P, Nisand I, Moreau L, et al. Controlled ovarian follicle puncture for egg retrieval. Transvaginal, sonographically. Lancet 1984 ; i : 1467 .

14. Belaisch-Allart JC, Frydman R, Testart J, et al. In vitro fertilization and embryo transfer program in Clamart, France. 7 In Vitro Fertil Embr Transf $1984 ; 1: 51-5$.

15. Gomel V. Recent advances in surgical correction of tubal diseases producing inf ertility. Curr Probl Obstet Gynecol 1978; I.

16. Edwards RG, Fishel SB, Cohen J, et al. Factors influencing the success of in vitro fertilization for alleviating human infertility. 7 In vitro Fertil Embr Transf 1984; $1:$ 3-23.

17. Quigley MM, Wolf DP. Human in vitro fertilization and embryo transfer at the University of Texas, Houston. 7 In Vitro Fertil Embr Transf 1984; 1 : 29-33.

18. Tyler JPP, Pryor JP, Collins WP. Heterologous ovum penetration by human spermatozoa. I Reprod Fertil 1981; $63: 499-508$.

19. Martin RH, Taylor PJ. Reliability and accuracy of the zona-f ree hamster ova assay in the assessment of male fertility. Br 7 Obstet Gynaeco 1982; 89 : 951-6.

20. Haas GG, Sokoloski JE, Wolf DP. The interfering effect of human IgG antisperm antibodies on human sperm penetration of zona-f ree hamster eggs. Am 7 Reprod Immunol 1980; 1 : 40-3.

21. Bronson RA, Cooper GW, Rosenfeld DL. Sperm-specific isoantibodies and autoantibodies inhibit the binding of human sperm to the human zona pellucida. Fertil Steril 1982; $38: 724-9$.

22. Ackerman SB, Graff D, Van Uem HM, et al. Immunologic infertility and in vitro fertilization. Fertil Steril 1984; $42: 474-7$.

23. Yovich JL, Stanger JD, Yovich JM, Turner SM, Newman BD. Treatment of male infertility by in vitro fertilization. Lancet 1984 ; ii : 169-70.

24. Muasher SJ, Garcia JE, Jones HW. Experience with diethylstilbestrol-exposed infertile women in a program of in vitro fertilization. Fertil

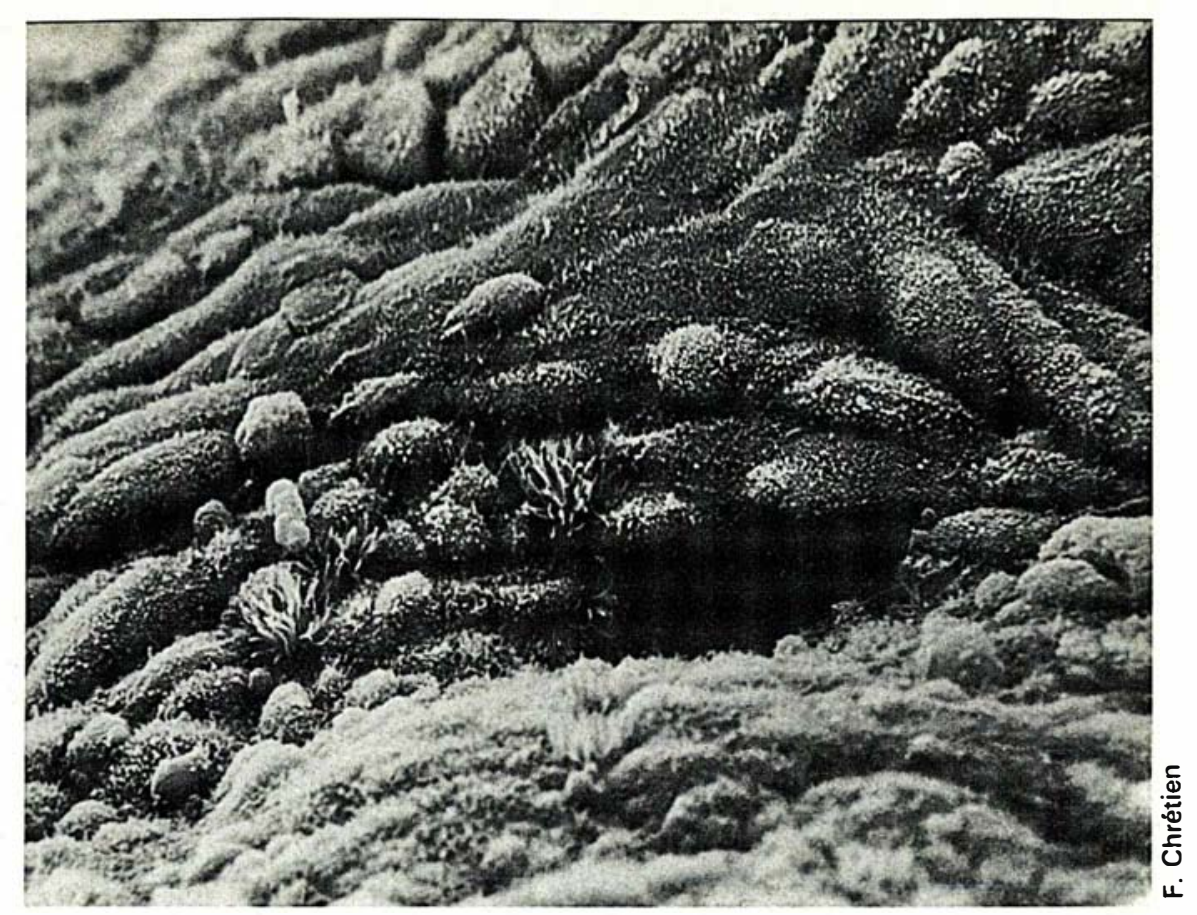

Epithélium de l'endomètre utérin.

ment employées dans le traitement de la stérilité féminine d'origine immunologique sont : l'emploi temporaire de préservatifs, l'immunosuppression par dérivés des corticoïdes et l'insémination intrautérine. Il a été démontré par le groupe de Norfolk [22] que le liquide folliculaire pouvait contenir des anticorps anti-spermatozoïdes, sans que cela altère la fécondation in vitro. Dans ces conditions, il est logique d'envisager la fécondation in vitro comme une possibilité de thérapeutique, encore faut-il comparer son efficacité avec d'autres méthodes telles que l'insémination intra-utérine avec préparation du sperme ou la fécondation naturelle. Chez l'homme, la présence d'autoanticorps anti-spermatozoïdes est une circonstance dans lequelle jusqu'ici, aucune thérapeutique n'avait été proposée; cependant Yovitch et coll. [23] ont montré que, dans ce cas, la fécondation in vitro pouvait être couronnée de succès, peut-être à cause du lavage des spermatozoïdes qu'implique cette technique.

\section{Stérilité idiopathique}

L'infertilité idiopathique concerne un couple qui cherche à avoir un enfant depuis plus de 2 ans, et dont les explorations classiques sont tout à fait normales. Trounson et Wood [5] ont recherché si certaines causes de stérilité idiopathique ne pouvaient pas relever d'un défaut de fécondation ou bien d'un défaut d'implantation. Les premiers résultats de la FIV ont montré dans ce cas des pourcentages de fertilisation supérieurs par rapport aux patientes ayant des trompes absentes; les données plus récentes montrent une stabilisation des résultats, quelle que soit l'indication, à partir du moment où le sperme est de bonne qualité. Dans ce groupe de patientes on peut inscrire la stérilité psychogène dont on ne sait si les troubles psychologiques précèdent l'installation de la période d'infertilité ou en sont une conséquence après une longue période d'attente.

\section{Endométriose}

La fécondation in vitro a été proposée en cas d'endométriose. Encore faut-il distinguer l'endométriose péritonéale diffuse de l'endométriose ovarienne. En effet dans le premier cas, et malgré le traitement spécifique, s'il persiste des foyers d'endométriose, on peut penser que la captation et la migration de l'ovocyte vers la trompe sera perturbée. La FIVETE permet de dépasser cet 
obstacle. Le taux de fertilisation et le taux de grossesse sont comparables à celui des autres groupes. La situation est différente lorsqu'il s'agit d'une endométriose ovarienne ; en effet, celle-ci réagit mal au traitement médical et la présence d'נJ:- kyste endométriosique va gêner la croissance des autres follicules et la collecte des ovocytes. Donc l'endométriose ovarienne ne constitue pas à nos yeux une bonne indication de la fécondation in vitro. Fnfin, de telles lésions peuvent siéger au niveau de l'utérus et on doit vérifier par hystéroscopie ou hystérographie l'existence ou non d'une adénomyose qui pourrait gêner l'implantation.

\section{Anomalie cervicale}

En cas d'absence de glaire, là encore afin de court-circuiter le passagé cervical, la fécondation in vitro est possible, mais elle est à mettre en concurrence avec l'insémination artificielle intra-utérine avec le sperme du conjoint.

Dans le cas de patientes présentant une cavité utérine anormale, conséquence de leur exposition au diéthylstilbestrol pendant la période intra-utérine, des auteurs américains [24] ont obtenu des résultats encourageants. Tout comme la tuberculose génitale, de telles situations semblaient jusqu'alors au-dessus de toute possibilité thérapeutique; la FIVETE off re ici de réelles possibilités.

Jusqu'à présent, nous avons envisagé la place de la fécondation in vitro au sein du couple, lorsqu'une anomalie venait gêner la fécondation naturelle. Cette technique, également, peut être utilisée avec des gamètes étrangères au couple.

\section{Le don d'ovules}

Les femmes qui pourraient bénéficier d'un don d'ovules sont celles dont les ovaires sont présents mais inaccessibles, rendant la fécondation in vitro trop dangereuse, voire impossible, ou les patientes dont les ovaires sont déficients (maladie génétique, endométriose ovarienne ou ménopause précoce), ou enfin les femmes dont les ovaires ont été retirés par intervention chirurgicale, mais qui conservent l'utérus.
En ce qui concerne la donneuse d'ovocytes, schématiquement quatre situations sont possibles :

la donneuse relationnelle, c'est-àdire la sour, l'amie intime qui est prête à subir cette mini-intervention pour donner un ou plusieurs ovocytes à une personne de son choix ;

la donneuse passionnelle qui ne connaitrait pas la receveuse, mais dont la motivation serait un pur altruisme;

la donneuse occasionnelle : une patiente accepterait en toute connaissance de causes de donner un ou plusieurs ovocytes au cours d'une intervention chirurgicale, ayant sa logique propre (ligature de trompes, colioscopie ou laparotomie);

la donneuse additionnelle: une patiente au cours d'une tentative de fécondation in vitro accepterait qu'un de ses ovules soit donné à partir d'un seuil numérique obtenu pour elle-même.

Une fois l'ovocyte obtenu, celui-ci peut être fécondé avec le sperme du couple infertile et il est soit réimplanté immédiatement chez la receveuse soit il est congelé afin d'être réimplanté ultérieurement. Dans le premier cas, cela nécessite une synchronisation des deux patientes, dans le deuxième cas, cela nécessite une maîtrise de la congélation.

La discussion éthique porte ici sur l'anonymat et la noncommercialisation des gamètes. Les dons d'ovules soulèvent de multiples problèmes sur le statut et le devenir des embryons obtenus. (Voir p. 154, l'entretien avec le Pr. David).

La fécondation in vitro aboutit, dans les meilleurs centres internationaux, à un enfant vivant dans I 2 à $15 \%$ des tentatives, alors que le taux de succès naturels avoisine les $25 \%$ par cycle. Il est possible qu'avec les développements de la stimulation ovarienne, la reconnaissance de la qualité embryonnaire et les techniques d'implantation, on puisse rejoindre, voire dépasser la nature. Dans l'état actuel de nos travaux, il nous semble indispensable d'évaluer l'efficacité de la fécondation in vitro face aux autres méthodes de traitement de la stérilité avant d'en faire la solution à chaque type d'infertilité

\section{Summary}

In vitro fertilization provides an increasingly effective response to the problems of sterility. Patients who can benefit from IVF treatment include those with the following problems: permanent tubal sterility; tubal sterility due to damaged tubes; certain malc sterility; idiopathic sterility; immunological sterility; pelvic endometriosis; nonexistent or hostile cervical mucus. The list of indications will grow as IVF encounters success in treating different problems.

\section{TIRÉS A PART}

R. Frydman : Hôpital Antoine-Béclère.157, ruc de la porte de Trivaux. 92I4I Clamart. 